\title{
End of Expected Battery Life
}

National Cancer Institute

\section{Source}

National Cancer Institute. End of Expected Battery Life. NCI Thesaurus. Code C99949.

The battery that supplies the device has reached the end of its life. (ACC) 